\title{
Risk Factors for Seizures in Children with Cerebral Palsy - A Descriptive Study
}

\author{
${ }^{1}$ B.Rajeshkannan, ${ }^{2}$ K.Ravikumar \\ ${ }^{1}$ Assistant Professor, ${ }^{2}$ Senior Resident, Dept. Of Paediatrics, KarpagaVinayaga Institute of Medical Science \& \\ Research Centre, Kanchipuram, TN, India.
}

\begin{abstract}
:
Background:

Children between the age group of 1 to 12 years suffering from cerebral palsy with and without seizures have a higher risk of mortality and morbidity. Few interventions likely to be beneficial like maternal risk factors, antenatal, natal, postnatal growth and development, neonatal seizures and its related complications ${ }^{l}$.

Aims and objectives: To find out the risk factors for Seizures in children with Cerebral Palsy.

To study maternal physical and socio-economic risk factors responsible for cerebral palsy and seizures in cerebral palsy also to identify significant modifiable risk factors for cerebral palsy in the locality, if any, and point out strategic measures to favourably modify those.

Materials and methods: It is a descriptive, Matched pair, case-control study. The study was done on Cerebral palsy babies in Karpaga Vinayaga Institute of Medical Science \& Research Centre, Kanchipuram, Tamilnadu during the period of January 2011-April 2016. This was a retrospective descriptive study of children with cerebral palsy (with and without seizures) were admitted. Obstetrical and maternal risk factors, natal and neonatal, growth and development upto the age of 12 years were collected. Overall 400 children were included in this study, Cerebral palsy children with and without seizures.

Results: Out of total 400children during 4years and 3 months (January 2011 - April 2016), there were 200 children had cerebral palsy without seizures and remaining children had cerebral palsy with seizures. The male population were $62.3 \%$ \& female were $37.7 \%$.

Conclusions: Maternal factors like Age, socio-economic status (SES), parity, height, pre-pregnancy weight, gestational weight gain, hypertension, anaemia, Previous history of IUGR, and adequacy of Anti-natal Checkup, maternal education, place of delivery, birth asphyxia, neonatal seizures, pattern of seizures, duration of seizures, number of episodes of seizures, family history of seizures, neonatal jaundice, were significant and to pinpoint modifiable ones \& suggest strategies to favourably modify cerebral palsy and seizures in cerebral palsy children.
\end{abstract}

Keywords: Cerebral Palsy, Maternal Risk Factors, Seizures.

\section{Introduction}

Cerebral Palsy is the most common neurological disability seen in children. The estimated prevalence of cerebral palsy in the developed countries is $2 / 1000$ population $^{3}$. The prevalence in a developing country like ours, is much higher, figures quoted being as high as 20 per 1000 live births. Epilepsy has been estimated to occur in $10-40 \%$ of cases, but its likelihood depends on the type of cerebral palsy ${ }^{3}$.

With a large population, the magnitude of the problem is enormous in India. This is a drain on the national economy, not only by means of the medical and other expenditure on these unfortunate children, but also in the form of loss of valuable human resources, which these youngsters would otherwise have contributed to the development of our nation ${ }^{2}$. Emotional sufferings and lost opportunities add immeasurably to the burden of the affected families. Hence, it is upon us to probe into the cause of this malady and to go all out to offer protection of our children from this developmental disaster and its complications ${ }^{4}$.

It is 150 years since Little's attribution of Cerebral Palsy to difficult deliveries and a century since Freud challenged it on the grounds of an underlying developmental disorder being the basic cause. The question is unanswered till date and the exact role of particular factors still debatable.

Knowledge of the etiology is important not only to protect the children from cerebral palsy but also from seizures as it is also significant in the light of present day consumerism and its medico-legal implications. 


\section{Methods}

It is a Descriptive; Matched pair, case-control study

Study Area: paediatric OPD of KarpagaVinayaga Institute of Medical Science \& Research Centre, Kanchipuram, Tamilnadu .

Study Population: Paediatric patients aged between 1 to 12 years.

Sample Size and Technique: Cerebral Palsy is a common disorder with an estimated prevalence of 2/1000 population in developed countries. The prevalence is much higher in developing countries, estimates showing as high as 20 per 1000 live births (2). Epilepsy $10-40 \%$ but its likelihood depends on the type of Cerebral Palsy. ${ }^{5}$

Data Collection:-The study was done on cerebral palsy in KarpagaVinayaga Institute of Medical Science \& Research Centre, Kanchipuram during the period of January 2011-April 2016. List of patients was obtained from the Op Register. Mothers of patients were interviewed to fill a printed structured questionnaire, after obtaining their consent. Certain clinical details were obtained from their OP cards and IP charts with patients consent. The collected data were filled up by the principal investigator on the study proforma. Controls too were selected from the OPD.

Inclusion Criteria: Cerebral Palsy children aged 1 to 12 yrs.

Exclusion Criteria: Suspected case of Neurodegenerative disorder. Family history of similar illness

Method of Data Analysis / Statistical Methods: Data collected were tabulated in a Microsoft ExcelTM data sheet and analysed.Prevalence of risk factors will be estimated in both the case and control to calculate odds ratio.

\section{Discussion}

There are several studies to find out the risk factors for Seizures in children with Cerebral Palsy. Overall 400 cases were included in this study, $63.2 \%$ were male population Rural population contributed to 252 (63.2\%), Urban population, Rural population contributed to home delivery and birth asphyxia. Maternal Factors Like place of delivery, Type of Delivery, Birth Asphyxia, and Multiple Birth.

Place of Delivery - Home delivery which is common in our country, especially when not attended by trained personnel has been identified as an important risk in the present study. Total Number of home delivery $44(11 \%)$. This is an area of high priority where all our maternal and child health services are directed towards, and still needs continued vigilance. Institutional Delivery - $356(89 \%)$ it included all the high risk pregnancy and the type of deliveries?.

Normal vaginal delivery is more in both case \& control 59.1\% (236). Prolonged labour is another important risk for $\mathrm{CP}$ in the present study. Caesarian sections 93 cases, control (59) 30.2\%, in cases 34 (17.3\%) Caesarian section are associated with high risk pregnancies, there should be expert management \& timely intervention to prevent Cerebral Palsy ${ }^{6}$.

Instrumental delivery by forceps or vacuum extraction constitutes a risk in the present study with forceps $19(4.8 \%)$ with increase in cases to $13(6.6 \%)$ with birth asphyxia. ${ }^{8}$ Although breech presentation was present only in 3 cases out of 200 it was also significant risk for seizure. Nearly one third of the CP cases who had breech presentation had an associated congenital malformation. It was postulated that it may be the result of an underlying developmental abnormality of the fetus ${ }^{10}$.

Birth Asphyxia - In the Present study birth asphyxia seen in 152 (38.3\%) of Study populationApgar scores as predictor of major neurological disability (26) found that low Apgar scores were significant risk factors for cerebral palsy, though $80 \%$ of them were free of any handicap. There was total number of $22(5.5 \%)$ multiple birth in both case and control. Neonatal Factors - Maturity \& Birth Weight. Low birth weight has been consistently and uniformly appreciated as an important risk and this is well reflected in the present study. 65 $(16.25 \%)$ cases were low birth weight babies. The increased association of maternal illiteracy with CP may be reflection of this. The present study shows that of the children with $\mathrm{CP}$ whose birth weight was known, belonged to the LBW category. This underlines the importance of the need for rigorous antenatal services and control of adverse antenatal factors identified to result in low birth weight infants. The increased risk of epilepsy in CP is believed to be linked to genetic and perinatal factors. First-degree relatives of children with $\mathrm{CP}$ and seizures have been reported to have an increased incidence of seizures. Among the perinatal factors, structural and developmental defects of the brain, chromosomal defects, intrauterine infections and hypoxic ischemic brain injuries are the more obvious causes that may result in seizures.

The objective of the study was to explore the relationship between cerebral palsy and epilepsy. Epilepsy is more common in certain types of $\mathrm{CP}$ than others with tetraplegic variety having the highest incidence of seizures (15 to 16\%) which in turn, might be a reflection of the severity of damage to the brain.

In the current study spastic tetraplegia was the commonest type of $\mathrm{CP}$ complicated by seizures. Spastic diplegia was the commonest type of CP in the control group of CP without seizures. Generalized tonic clonic type of seizures was more common. Epilepsy was found to have an earlier age of onset, poorer seizure control, 
increased risks of status epilepticus and a need for more than one antiepileptic drug for seizure control in children withCP and seizures.

There was also a higher incidence of neonatal seizures in this group of children as compared with control group. This possibly reflects a more severe birth asphyxia. In particular, children with spastic tetraplegia more often needed polytherapy. This may be due to the more extensive brain pathology seen on brain imaging in this group of children. It is also possible that certain genetic factors, as yet unidentified, may play a role in the pathogenesis of seizures in children with $\mathrm{CP}$, particularly in the tetraplegic variety. When we compare the two groups of children with $\mathrm{CP}$ with seizures and those without seizures. The following variables seem to have an increased association with the seizures group: history of birth asphyxia, term delivery, a history of neonatal seizures, spastic tetraplegic variety of $\mathrm{CP}$, history of seizures in first year of life, type of seizures and presence of abnormal CT findings.

\section{Conclusions}

1. Risk factor for Seizures in children with cerebral palsy were rural population, low socio economic status, home delivery, low birth weight, preterm, birth asphyxia and multiple births.

2. All the risk factors which are identified to be significant are preventable by an efficient health care delivery system and good health education strategies. These are the area of high priority.

3. Health education to reduce early marriage and pregnancy and to avoid home delivery will help to eliminate the risk factors. Good antenatal care will reduce the incidence of low birth weight. Improvement of obstetric care and effective management of prolonged labour and difficult labour will also help to reduce the problem of birth asphyxia and complications of multiple births.

4. Congenital malformation of Central Nervous System by CT brain/MRI are being commonly, identified in both the case and control in the present study $(26.4 \%, 10.4 \%)$ respectively. Periventricular leucomalacia is more in spastic diplegia.

5. Seizures are more common in certain types of cerebral palsy than other with Spastic quadriplegia variety having the highest incidence of seizures ranging from $40-60 \%$ which in turn might be a reflection of the severity of damage of the brain. Spastic quadriplegia being the most common type in this study with spastic diplegia in the control.

6. Generalized tonic clonic seizures, multiple episodes, lasting more than 5 - 30 minutes with more than 2-6 episodes per year, requiring polytherapy is found to be common in this Study.

\section{References}

[1] Hadjipanayis, A., Hadjichristodoulou, C., Youroukos, S. Epilepsy in patients with cerebral palsy. Dev Med Child Neurol. 1997;39:659-663.

[2] Kwong, K., Wong, S.N., So, K. Epilepsy in children with cerebral palsy. PaediatrNeurol. 1998;19:313-316.

[3] Zafeiriou, D.I., Kontopoulos, E.E., Tsikoulas, I. Characteristics and prognosis of epilepsy in children with cerebral palsy. J Child Neurol. 1999;14:289-294.

[4] Wallace, S.J. Epilepsy in cerebral palsy. Dev Med Child Neurol. 2001;43:713-717

[5] Gururaj, A.K., Sztriha, L., Bener, A., Dawodu, A., Eapen, V. Epilepsy in children with cerebral palsy.Seizure. 2003;12:110-114.

[6] Singhi, P., Jagirdar, S., Khandelwal, N., Malhi, P. Epilepsy in children with cerebral palsy. J Child Neurol. 2003;18:174-179.

[7] Kulak, W., Sobaniec, W. Risk factors and prognosis of epilepsy in children with cerebral palsy in north-eastern Poland. Brain Dev. 2003;25:499-506.

[8] Ronen, G.M., Buckley, D., Penney, S., Streiner, D.L. Long-term prognosis in children with neonatal seizures: a population-based study. Neurology. 2007;69:1816-1822.

[9] Sun, Y., Vestergaard, M., Pedersen, C.B., Christensen, J., Olsen, J. Apgar scores and long-term risk of epilepsy. Epidemiology. 2006; 17:296-301.

[10] Krebs, L., Langhoff-Roos, J. The relation of breech presentation at term to epilepsy in childhood.Eur J ObstetGynecolReprod Biol. 2006;127:26-28.

[11] Eherenstein, V., Pedersen, L., Holsteen, V., Larsen, H., Rothman, K.J., Sørensen, H.T. Postterm delivery and risk for epilepsy in childhood. Pediatrics. 2007;119:554-561.

[12] Proposal for revised classification of epilepsies and epileptic syndromes. Commission on classification and terminology of the international league against epilepsy. Epilepsia. 1989;30:389-399.

[13] Usher, R., McLean, F. Intrauterine growth of live-born Caucasian infants at sea level: standards obtained from measurements in 7 dimensions of infants born between 25 and 44 weeks gestation. J Pediatr. 1969;74:901-910.

[14] Cowan, L.D., Bodensteiner, J.B., Leviton, A., Doherty, L. Prevalence of the epilepsies in children and adolescents. Epilepsia. 1989; 30:94-106.

[15] Krageloh-Mann, I., Hagberg, G., Meisner, C., Schelp, B., Haas, G., Eeg-Olofsson, K.E. et al, Bilateral spastic cerebral palsy - a comparative study between south-west Germany and western Sweden. I: clinical patterns and disabilities. Dev Med Child Neurol. 1993; 35:1037-1047. 